\title{
A osteonecrose dos maxilares induzida por bifosfonatos: uma revisão de literatura
}

\author{
Bisphosphonate-induced osteonecrosis of the jaws: a literature review \\ Osteonecrosis de los maxilares inducida por bisfosfonatos: revisión de la literatura
}

Recebido: 17/11/2021 | Revisado: 25/11/2021 | Aceito: 26/11/2021 | Publicado: 07/12/2021

\author{
Maria Caroline Dorigan \\ ORCID: https://orcid.org/0000-0003-2750-9053 \\ Universidade São Francisco, Brasil \\ E-mail: carolinedorigan@ hotmail.com \\ Juliano Bernardes Matias \\ ORCID: https://orcid.org/0000-0003-3961-2509 \\ Universidade São Francisco, Brasil \\ E-mail: jbmatias123@gmail.com \\ Valdinéia Maria Tognetti \\ ORCID: https://orcid.org/0000-0002-9150-1980 \\ Universidade São Francisco, Brasil \\ E-mail: valdineia.tognetti@usf.edu.br \\ Silva Cristina Mazeti Torres \\ ORCID: https://orcid.org/0000-0001-9128-5790 \\ Universidade São Francisco, Brasil \\ E-mail: silvia.torres@usf.edu.br
}

\begin{abstract}
Resumo
Os bifosfonatos atuam no processo de remodelação óssea, impedindo a reabsorção patológica ocasionada por determinadas doenças como a osteoporose, osteogênese imperfeita, doença de Paget e neoplasias com metástases ósseas. O bifosfonato incorpora na matriz óssea devido a sua alta afinidade com os ossos, caracterizando seu efeito cumulativo e proporcionando o risco de desenvolvimento da osteonecrose dos maxilares induzida por bifosfonatos, que discorre de uma complicação decorrente do uso desses fármacos onde existe a exposição óssea necrótica patológica de ossos maxilares podendo ser de surgimento espontâneo ou induzida por intervenções odontológicas. O objetivo deste estudo visa a compreensão da osteonecrose e sua etiologia afim de identificar fatores de risco locais ou sistêmicos, métodos de prevenção, evidenciar meios de diagnóstico através de sinais e sintomas clínicos e exames complementares além da realização do seu estadiamento em caso de ocorrência e os respectivos tratamentos existentes. Para a construção da revisão de literatura alguns passos foram determinantes como a elaboração de questões norteadoras e hipóteses, pesquisa bibliográfica para coleta de dados seguindo critérios de inclusão e exclusão através de análise minuciosa e interpretação de resultados. Com o envelhecimento populacional o número de pessoas usando medicações antirreabsortivas aumentou significativamente e com isso, o risco de desenvolvimento de complicações como a abordada também cresceu. Conclui-se que é de extrema importância o papel do cirurgiãodentista em uma equipe multidisciplinar para tratamento destes indivíduos que fazem uso deste tipo de fármaco afim de evitar e diminuir a ocorrência da osteonecrose garantindo assistência, saúde e bem-estar ao paciente.
\end{abstract}

Palavras-chave: Osteonecrose; Bifosfonatos; Maxilares; Conservadores da densidade óssea.

\begin{abstract}
Bisphosphonates act in the bone remodeling process, preventing pathological resorption caused by certain diseases such as osteoporosis, osteogenesis imperfecta, Paget's disease and neoplasms with bone metastases. The bisphosphonate incorporates into the bone matrix due to its high affinity with bone, characterizing its cumulative effect and providing the risk of developing osteonecrosis of the jaws induced by bisphosphonates, which is a complication arising from the use of these drugs where there is a pathological necrotic bone exposure of jawbones, which may arise spontaneously or be induced by dental interventions. The aim of this study is to understand osteonecrosis and its etiology to identify local or systemic risk factors, prevention methods, and to show means of diagnosis through clinical signs and symptoms and complementary exams, in addition to staging it in case of occurrence and the respective existing treatments. For the construction of the literature review some steps were determinant as the elaboration of guiding questions and hypotheses, literature search for data collection following inclusion and exclusion criteria through detailed analysis and interpretation of results. With the aging of the population, the number of people using antiresorptive medications has increased significantly, and with this, the risk of developing complications such as this one has also increased. We conclude that the role of the dental surgeon in a multidisciplinary team is extremely important for the treatment of these individuals who use this type of drug to prevent and reduce the occurrence of osteonecrosis, guaranteeing assistance, health, and well-being to the patient.
\end{abstract}

Keywords: Osteonecrosis; Diphosphonates; Jaws; Bone density conservation agents. 


\begin{abstract}
Resumen
Los bifosfonatos actúan en el proceso de remodelación ósea, impidiendo la reabsorción patológica causada por ciertas enfermedades como la osteoporosis, la osteogénesis imperfecta, la enfermedad de Paget y las neoplasias con metástasis óseas. El bifosfonato se incorpora en la matriz ósea debido a su alta afinidad con los huesos, caracterizando su efecto acumulativo y proporcionando el riesgo de desarrollar osteonecrosis de los maxilares inducida por los bifosfonatos, que es una complicación resultante del uso de estos fármacos donde hay una exposición ósea necrótica patológica de los maxilares, que puede ser espontánea o inducida por intervenciones dentales. El objetivo de este estudio es conocer la osteonecrosis y su etiología para identificar los factores de riesgo locales o sistémicos, los métodos de prevención, los métodos de diagnóstico a través de los signos y síntomas clínicos y los exámenes complementarios, así como su estadificación en caso de aparición y los respectivos tratamientos existentes. Para la construcción de la revisión bibliográfica fueron determinantes algunos pasos como la elaboración de preguntas orientadoras e hipótesis, la investigación bibliográfica para la recopilación de datos siguiendo los criterios de inclusión y exclusión mediante el análisis detallado y la interpretación de los resultados. Con el aumento de la población, el número de personas que utilizan medicamentos anti-reabsorción aumentó significativamente y, con ello, también creció el riesgo de desarrollo de complicaciones como la abordada. Se concluye que es de extrema importancia el papel del cirujano-dentista en un equipo multidisciplinar para el tratamiento de los individuos que hacen uso de este tipo de fármaco con el fin de evitar y disminuir la aparición de la osteonecrosis garantizando la asistencia, la seguridad y el bienestar del paciente.
\end{abstract}

Palabras clave: Osteonecrosis; Bifosfonatos; Tiburón; Conservadores de la densidad ósea.

\title{
1. Introdução
}

Algumas patologias como Osteoporose, Doença de Paget, neoplasias com ou sem metástases ósseas, interferem no metabolismo ósseo, proporcionando um desequilíbrio no processo de remodelação que resulta em uma reabsorção exagerada ocasionando diminuição da quantidade e qualidade dos ossos (Bicudo, 2017; Fernandes et al., 2005; Sousa \& Jardim Junior, 2008; Mendes, 2017). Diante deste cenário, alguns medicamentos antirreabsortivos surgiram com a finalidade de diminuir essa reabsorção patológica (Castro et al., 2004; Fernandes et al.,2005; Sousa et al., 2008; Mendes, 2017).

É o caso dos Bifosfonatos análogos sintéticos de uma substância endógena (pirofosfato) que garante a homeostasia durante o processo de remodelação em situações fisiológicas. Esses fármacos atuam diretamente nos osteoclastos promovendo sua apoptose e consequente diminuição da atividade osteoclástica (Castro et al., 2004; Fernandes et al, 2005; Chaves et al., 2018; Mendes, 2017; Santos \& Ferreira Neto, 2021; Carlessi \& Maragno, 2017; Parrião et al., 2021). O efeito adverso desses medicamentos de maior importância para a área odontológica é a Osteonecrose dos maxilares.

A Osteonecrose dos maxilares induzida por bifosfonatos é caracterizada pela exposição do osso necrótico por mais de 8 semanas em região de maxila e/ou mandíbula, em pacientes com histórico do uso de bifosfonatos ou outros antirreabsortivos, sem histórico de neoplasia em região de cabeça e pescoço (Ruggiero et al., 2009; Ruggiero et al., 2014). O quadro clínico pode também apresentar: sintomatologia dolorosa, tumefação em região circundante e mucosa, fraturas patológicas e manifestação de infecções (Bicudo, 2017; Ruggiero et al., 2009; Ruggiero et al., 2014; Couto, 2018). Possui etiologia multifatorial e apesar de pouco elucidada, algumas hipóteses e fatores são levados em consideração na tentativa de justificar como e porque a complicação acontece (Bicudo, 2017; Couto, 2018).

O risco de desenvolvimento da Osteonecrose dos Maxilares pode variar de acordo com a via e tempo de administração, tipo de fármaco e dosagem. Além disso, fatores de risco locais como traumas em tecidos moles e fatores de risco sistêmicos associados a presença de comorbidades por exemplo, estão diretamente ligados ao maior risco de incidência desta complicação (Bicudo, 2017; Castro et al., 2004; Fernandes et al., 2005; Chaves et al., 2018; Ruggiero et al., 2009; Ruggiero et al., 2014; Couto, 2018; Sousa et al., 2008; Fahd, 2020; Santos et al., 2021; Pinto Junior et al., 2017; Carlessi et al., 2017; Santos et al., 2020).

Diante disso, é de extrema importância para o cirurgião-dentista: estar atento à saúde geral do seu paciente, em especial sobre todo e qualquer tratamento de saúde realizado previamente, concomitantemente e posteriormente ao tratamento odontológico; aos sinais e sintomas clínicos a fim de realizar a prevenção e em casos de ocorrência, o correto diagnóstico e 
consequente tratamento da osteonecrose, evitando sua progressão. A despeito da severidade da complicação, o presente estudo visa a compreensão da Osteonecrose dos maxilares induzida por bifosfonatos bem como entendimento de critérios e meios para diagnóstico, prevenção e tratamento.

\section{Metodologia}

O presente estudo se caracteriza como uma revisão de literatura sobre a osteonecrose dos maxilares induzida por bifosfonatos e a importância do diagnóstico e prevenção desta complicação. A amplitude e atualidade do tema no meio odontológico permite o detalhamento minucioso sobre diferentes abordagens atuais para diagnóstico, prevenção e tratamento da osteonecrose.

Diante disso, foram estabelecidas etapas para a construção desta revisão de literatura, sendo: elaboração de questões norteadoras, busca em literatura e coleta de dados seguindo critérios de inclusão e exclusão, análise dos estudos selecionados, interpretação dos resultados e elaboração da revisão de literatura propriamente dita. Como instrumento de coleta de dados, foi realizada busca em base de dados como: Google acadêmico, Pubmed, Scielo, ScienceDirect e outros fazendo uso de palavraschave como por exemplo: "Osteonecrose dos maxilares", "osteonecrose induzida por medicamentos", "bifosfonatos", "tratamento da osteonecrose dos maxilares". Foi realizada busca bibliográfica em livros - A Terapia com Bifosfonatos e a Osteonecrose dos Maxilares de Elis Zéfiro Couto. Aulas, cursos e palestras com especialistas também contribuíram com a busca em base de dados sendo de suma importância para a construção deste estudo.

Os artigos selecionados foram aqueles que após leitura e análise de atualidade (considerando ano de publicação, autores, abordagens atuais e outros requisitos) se enquadram no enfoque do trabalho e foram excluídos aqueles que se não se enquadravam e/ou apresentavam apenas seu resumo disponível.

\section{Resultados}

O pirofosfato é uma substância endógena que atua como regulador fisiológico da calcificação e remodelação óssea diante de situações fisiológicas garantindo o equilíbrio entre deposição e reabsorção óssea. O pirofosfato sofre hidrólise acelerada pelas enzimas da mucosa gastrointestinal o que resultou na necessidade de desenvolvimento de um fármaco com maior resistência a degradação enzimática, para uso diante de situações de desequilíbrio no processo de remodelação que algumas doenças proporcionam. Neste contexto surgiram os bifosfonatos, análogos sintéticos do pirofosfato onde o átomo central de oxigênio é substituído por um átomo de carbono tornando-os além de mais resistentes a degradação enzimática, tornam sua meia-vida aumentada e com capacidade de influenciar efetivamente o metabolismo ósseo (Castro et al., 2004; Fernandes et al., 2005; Chaves et al., 2018; Mendes, 2017).

Esses fármacos são amplamente utilizados desde 1960 e agem ligando-se às zonas de reabsorção óssea diminuindo a atividade osteoclástica e proporcionando consequente alteração no processo de remodelação óssea (Castro et al., 2004; Fernandes et al., 2005; Chaves et al., 2018; Sousa et al., 2008; Mendes, 2017; Santos et al., 2021; Pinto Junior et al., 2017; Carlessi et al., 2017). Podem ser classificados ainda em: não nitrogenados ou de $1^{a}$ geração, quando inativam os osteoclastos a partir da formação de metabólitos citotóxicos análogos da adenosina trifosfato (ATP). O bifosfonato então, devido à sua alta afinidade, liga-se aos minerais do osso e é absorvido pelos osteoclastos à medida que o osso é reabsorvido. Seu poder acumulativo, interfere nas enzimas metabólicas intracelulares provocando a apoptose das células osteoplásticas (Chaves et al., 2018; Mendes, 2017).

Já os nitrogenados, ou de segunda geração chegam a ser de 10 a 1000 vezes mais potentes devido a sua maior afinidade com a molécula de nitrogênio com cálcio presentes no tecido ósseo e seu mecanismo baseia-se na inibição de farnesil pirofosfatase uma enzima que permite a formação de metabólitos essenciais para a prenilação de proteínas (adição de 
moléculas hidrofóbicas) na via intracelular do mevalonato e na formação do colesterol como seu subproduto. Essa prenilação ou lipidação permite a ligação de pequenas proteínas à membrana celular ocorrendo a apoptose dos osteoclastos pela quebra da borda em escova dos mesmos (Chaves et al., 2018; Mendes, 2017).

Entre os principais efeitos adversos, destacados estão: complicações gastrointestinais como úlceras e intolerância, falência renal, hipocalcemia, mialgia, transtornos inflamatórios oculares, fibrilação auricular, e a de maior relevância em termos de gravidade e com maior importância para a odontologia destaca-se a osteonecrose dos maxilares (Mendes, 2017; Fahd, 2020).

Os primeiros 36 casos relatados em literatura foram realizados no ano de 2003 onde foi relatada a exposição óssea dolorosa em ossos maxilares sem resposta a terapia cirúrgica e tratamentos em pacientes oncológicos em uso de medicamentos antirreabsortivos (Pamidronato e o Zolendronato) alertando ainda para o risco de "epidemia em crescimento" (Marx, 2003).

Em 2005 determinou-se que a exposição óssea dolorosa relatada anteriormente tratava-se de uma osteonecrose caracterizada com uma complicação advinda do uso de bifosfonatos dando origem à sigla BRONJ (Bisphosphonate-Related Osteonecrosis of the Jaw) em português: Osteonecrose dos maxilares induzida por bifosfonatos (Bicudo, 2015; Ruggiero et al., 2009; Couto, 2018). Em 2014 a AAOMS (American Association of Oral and Maxillofacial Surgeons) publica uma atualização de um position paper (documento com o objetivo de apresentar um posicionamento) publicado no ano de 2007 (Ruggiero et al., 2009) atualizando a sigla para MRONJ (Medication-related ostheonecrosis of the jaw) em português OMRM (Osteonecrose dos maxilares induzida por medicamentos) acrescentando além dos Bifosfonatos os Denosumabes e Drogas Antiangiogênicas devido ao fato de que essas também estão relacionadas ao surgimento desta complicação (Bicudo, 2015; Ruggiero et al. 2014).

A etiologia da osteonecrose dos maxilares ainda não é completamente elucidada, mas acredita-se que alguns fatores influenciam diretamente no surgimento desta complicação, tais como: a elevada supressão do turnover ósseo (Santos et al., 2021; Pinto Junior et al., 2017; Parrião et al., 2021) em pacientes que utilizam bifosfonatos em regiões de ossos maxilares que devido a movimentos mastigatórios e fala requerem um alto índice de remodelação óssea. Além disso o efeito antiangiogênico das drogas diminuem a vascularização e consequente irrigação sanguínea dos tecidos ósseos e tecidos moles causando a hipóxia e morte destes (Spezzia, 2019; Carneiro et al., 2020). Este fator justifica inclusive o fato da osteonecrose acometer mais a região de mandíbula devido a vascularização dessa região ser menor em relação à maxila. Além disso, o efeito antiangiogênico afeta diretamente na toxicidade dos tecidos moles que se tornam mais fibrosos e menos elásticos devido a diminuição da vascularização destes, tornando-os mais susceptíveis a microtraumas expondo a região óssea dos maxilares que é protegida apenas por esta fina camada e mucosa (Bicudo, 2015; Ruggiero et al., 2014; Couto, 2018; Sousa et al., 2008).

Em uma atualização de 2014 realizada pela AAOMS sobre a osteonecrose dos maxilares, a associação cita como critérios de diagnóstico: área de exposição óssea ou osso que possa ser sondado através de fístula intra ou extraoral de surgimento espontâneo ou induzido por cirurgias em região maxilofacial que persista por 8 ou mais semanas; tratamento atual ou prévio com medicações antirreabsortivas ou antiangiogênicas e ausência de histórico de radioterapia em região maxilofacial e de doença metastática óbvia na região dos maxilares (Ruggiero et al., 2014).

Existem situações de rotina clínica como doenças periodontais, abcessos faciais, lesões periapicais com fístula, trismo, parestesia entre outras que trazem sinais e sintomas semelhantes à osteonecrose, então é preciso conhecer as patologias que fazem diagnóstico diferencial para que não haja diagnóstico incorreto e posterior evolução da osteonecrose (Bicudo, 2015). Potência, dosagem, tempo de uso e via de administração também possuem grande influência na indução desta complicação (Santos et al., 2021; Pinto Junior et al., 2017; Carlessi et al., 2017; Santos, et al., 2020).

Diante disso a AAOMS realizou o estadiamento da osteonecrose dos maxilares (Tabela 1) a partir dos sinais e sintomas clínicos presentes a fim de auxiliar no diagnóstico correto da osteonecrose e impor um protocolo de tratamento de 
acordo com os vários estágios. O paciente em risco de desenvolvimento seria aquele faz ou já fez uso de bifosfonatos via oral ou endovenosa, mas que não apresenta osso necrótico exposto ou aparente. Já o estágio 0 é descrito pela ausência de sinais clínicos de osso necrótico, mas, com achados clínicos específicos e presença de alterações radiográficas e sintomas.

Tabela 1 - Estágios da Osteonecrose segundo a AAOMS.

\begin{tabular}{ll}
\hline ESTÁGIO & DESCRIÇÃO \\
\hline Em risco & Sem osso necrótico aparente em pacientes que têm sido tratados com bifosfonatos orais ou intravenosos. \\
Estágio 0 & Ausência de sinais clínicos de osso necrótico, mas com achados clínicos inespećficos, alterações radiográficas e sintomas. \\
Estágio 1 & $\begin{array}{l}\text { Fístulas ou ossos necróticos expostos, com ausência de sintomatologia e infeção. } \\
\text { Estágio 2 }\end{array}$ \\
$\begin{array}{l}\text { Fístula ou ossos necróticos expostos, com presença de sintomatologia e infeção - com ou sem drenagem purulenta e presença } \\
\text { de eritema. }\end{array}$ \\
$\begin{array}{l}\text { Exposição óssea necrótica com extensão além do osso alveolar (borda inferior e ramo da mandíbula, seio maxilar, arco } \\
\text { zigomático) com presença de sintomatologia dolorosa e associada à infecção, podendo resultar em fratura patológica e } \\
\text { comunicação buco sinusal. }\end{array}$ \\
\hline
\end{tabular}

Fonte: Ruggiero et al. (2014) (adaptado).

O paciente em estágio 1 apresenta fístula ou osso necrótico exposto, com ausência de sintomatologia e infecção (Figura 1), o que o difere do estágio 2 (Figura 2) que apresenta sintomatologia dolorosa e infecção com ou sem drenagem purulenta e presença de eritema.

Figura 1 - Estágio 1 da Osteonecrose dos maxilares.

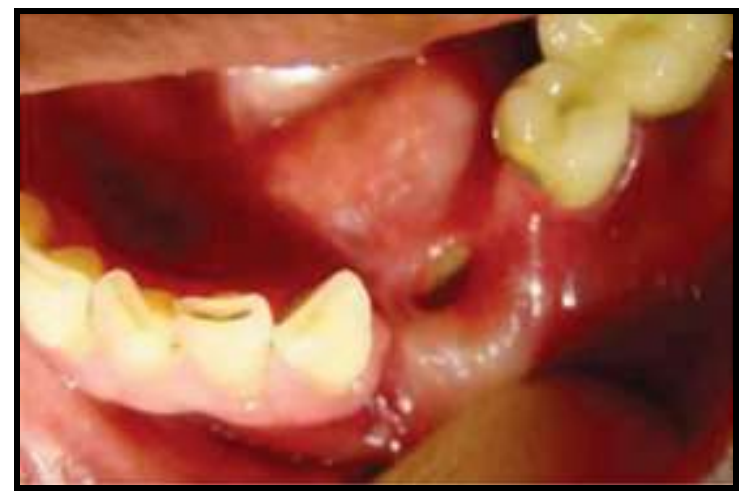

Fonte: Brozoski et al. (2012).

Figura 2 - Estágio 2 da Osteonecrose dos maxilares.

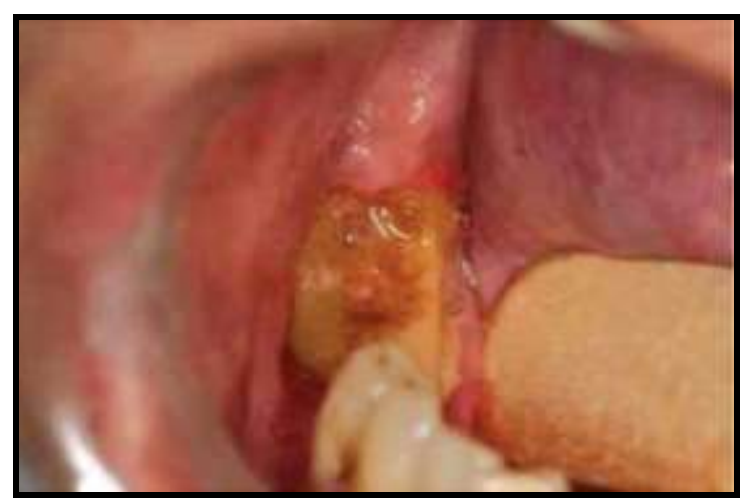

Fonte: Brozoski et al. (2012). 
No estágio 3, a exposição óssea necrótica se estende além do osso alveolar (borda inferior e ramo da mandíbula, seio maxilar, arco zigomático) e apresenta sintomatologia dolorosa e associada a infecção, podendo resultar em fratura patológica e comunicação buco sinusal (Figura 3).

Figura 3 - Estágio 3 da Osteonecrose dos maxilares.

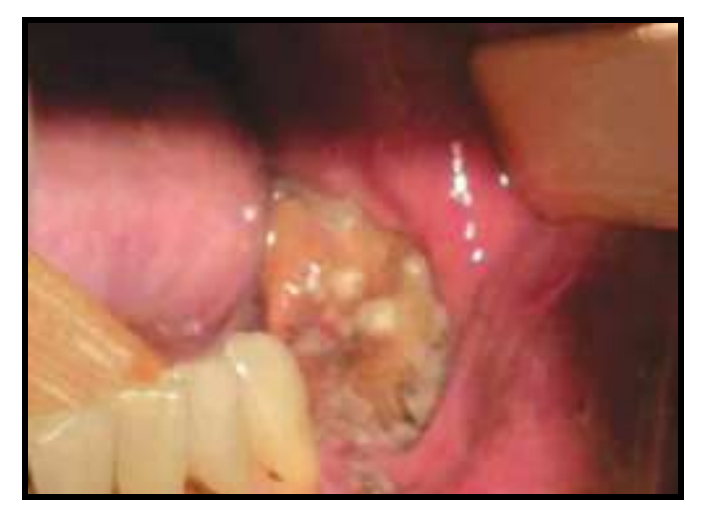

Fonte: Brozoski et al. (2012).

Devido ao comprometimento ósseo decorrente da osteonecrose, exames imaginológicos como Radiografias Panorâmicas, Tomografias Computadorizadas e Tomografias Computadorizadas de Feixe Cônico são amplamente utilizados para auxiliar no correto diagnóstico, orientação, determinação do estadiamento das lesões (Carneiro et al., 2020; Oliveira et al., 2020; Meneguini et al., 2017; Pexe, Curi, Costa, Koga \& Cardoso et al., 2018).

As principais alterações decorrentes da osteonecrose dos maxilares citadas por estudos em exames radiográficos panorâmicos são: presença de imagens radiolúcidas difusas em região maxilo-mandibular, aumento do espaço do ligamento periodontal em especial em regiões de furca, presença de espaços alveolares crônicos ocasionados por alterações escleróticas que acometem a lâmina dura dos alvéolos após extrações dentárias, sendo muito comum a presença de esclerose óssea e o comprometimento de lâmina dura em estágios mais avançados da doença (Bicudo, 2015; Olutayo et al., 2010; Arce et al., 2009; Spezzia, 2019).

Neste tipo de exame, temos como principal vantagem a amplitude desta radiografia por nos oferecer uma imagem de todo o complexo maxilo-mandibular e como desvantagem o fato de ser um exame bidimensional dificultando a diferenciação de demais hipóteses diagnósticas (como osteorradionecrose, metástases e Doença de Paget) devido a impossibilidade de estabelecer os limites da lesão, mostrando-se mais eficaz em estágios mais avançados da doença, evidenciando-se então, a importância da Tomografia Computadorizada e Tomografia Computadorizada de Feixe Cônico, exames imaginológicos tridimensionais caracterizando sua principal vantagem, oferecendo imagens com maior definição e possibilitando a identificação e definição das margens da lesão determinando sua extensão e consequentemente sua gravidade mesmo em estágios iniciais (Bicudo, 2015; Olutayo et al., 2010).

Podem ser observadas também alterações além das já citadas acima como: neoformação óssea e presença de sequestros ósseos. É valido salientar que estágios iniciais como o estágio 1, apresentam alterações discretas à nível periapical, devido à limitação do estágio inicial podendo ser solicitado uma TCFC ou até mesmo, é possível identificar tais alterações em radiografias periapicais em alguns casos (Olutayo et al., 2010; Meneguini et al., 2017).

A solicitação de exames laboratoriais tais como o CTX-1 (marcador sérico de reabsorção óssea) tem por finalidade de avaliar os níveis séricos desses marcadores que podem sugerir (não definir) a ocorrência ou não de osteonecrose diante de uma intervenção cirúrgica em pacientes usuários de bifosfonatos ou outras medicações antirreabsortivas. Neste exame o telopeptídeo carboxiterminal de ligação cruzada do colágeno 1 (prevalente no osso) é detectado e quantificado em exames de 
sangue ou urina que devem ser realizados em jejum preferencialmente devido a alimentação ser um fator que pode alterar o resultado do exame.

A unidade de medida utilizada é o picograma por mililitro ( $\mathrm{pg} / \mathrm{ml})$, sendo que: durante a terapia com antirreabsortivos temos valores de CTX-1 inferiores a $100 \mathrm{pg} / \mathrm{ml}$ até $150 \mathrm{pg} / \mathrm{ml}$ indicando risco elevado e moderado, respectivamente. Acima de $150 \mathrm{pg} / \mathrm{ml}$ o risco é baixo de desenvolvimento de osteonecrose indicando reabsorção óssea aumentada (Bicudo, 2015; Chaves et al., 2018; Couto, 2018; Lazarovici et al., 2010) Porém, estudos recentes revelaram que o CTX-1 não se mostrou um exame não muito eficaz para avaliar risco de desenvolvimento da osteonecrose uma vez que esses marcadores podem ser facilmente alterados pelo uso dos bifosfonatos ou pela própria doença em tratamento (Bicudo, 2015; Dal-Prá et al., 2016).

A prevenção da osteonecrose está diretamente ligada na adequação de meio bucal que antecede a terapia com os bifosfonatos e na avaliação de risco de desenvolvimento da osteonecrose nos casos em que o paciente já iniciou a terapia (Bicudo, 2015; Sturrock et al., 2019).

O cuidado odontológico preventivo antes e durante a terapia com bifosfonatos diminui em cerca de $50 \%$ o risco de desenvolvimento da osteonecrose dos maxilares e que equivalem por exemplo ao preparo de um paciente submetido a um procedimento cirúrgico cardíaco (Bicudo, 2015; Wan et al., 2020).

A avaliação de risco individual envolve o conhecimento do cirurgião-dentista de fatores relacionados aos medicamentos, tais como: as principais drogas relacionadas ao risco de desenvolvimento da osteonecrose dos maxilares, seu mecanismo de ação, indicações dessas medicações e capacidade de incorporação óssea, por exemplo. Além disso, uma anamnese bem detalhada permite ao profissional o acesso de informações indispensáveis como as vias de administração (oral ou endovenosa), tempo, dose, potência da medicação e frequência de uso, datas da última e da próxima administração. Outros fatores estão relacionados à saúde do paciente e podem ser de origem sistêmica, já que algumas comorbidades como a diabetes e pacientes em tratamento quimioterápico tem sua cicatrização dificultada por conta dessas condições, elevando consequentemente o risco de osteonecrose. $\mathrm{O}$ uso de alguns medicamentos como antitrombóticos, anticoagulantes também podem elevar o risco por interferirem na cicatrização (Bicudo, 2015).

A realidade ideal seria aquela em que o paciente iniciasse o tratamento com bifosfonatos após controle destes fatores cabendo ao cirurgião-dentista realizar o acompanhamento periódico desses pacientes, previamente através da adequação de meio bucal, eliminação de focos de infecção e eliminação de fontes de trauma; concomitantemente com a manutenção da saúde bucal com tratamento odontológico periódico lembrando de não realizar intervenções invasivas sempre que possível e correto manejo da osteonecrose diante de sua ocorrência; e após o uso dos bifosfonatos mantendo a saúde bucal e realizando mapeamento de risco após o térmico da medicação (Bicudo, 2015; Sturrock et al., 2019; Gonzales \& Azevedo, 2019; Spezzia, 2019; Carneiro et al., 2020; Meneguini et al., 2017).

O tratamento da osteonecrose dos maxilares visa sempre o fechamento da ferida necrótica. É de difícil realização, requer a associação de várias terapias e propõe o controle da dor, infecção e progressão da osteonecrose. Para isso, é necessário realizar classificação individual de risco, estadiamento da osteonecrose e avaliar as condições sistêmicas atuais do paciente (impossibilidade por exemplo de realizar tratamentos cirúrgicos em pacientes quimioterápicos ou alérgicos à determinada medicação) (Bicudo, 2015; Ruggiero et al., 2014; Oliveira et al., 2014; Vilela-Carvalho et al., 2018).

Para isso a AAOMS definiu um protocolo de condutas recomendadas que variam de acordo com o estágio da osteonecrose. (Tabela 2) 
Tabela 2 - Classificação de risco da osteonecrose dos maxilares e conduta recomendada.

\begin{tabular}{|c|c|c|}
\hline ESTÁGIO & DESCRIÇÃO & CONDUTA \\
\hline Em risco & $\begin{array}{l}\text { Sem evidência clínica de osso necrótico, ausência de achados } \\
\text { radiográficos em pacientes que fizeram uso de bifosfonatos orais ou } \\
\text { endovenosos (drogas antirreabsortivas e/ou antiangiogênicas). }\end{array}$ & $\begin{array}{l}\text { Orientações ao paciente; acompanhamento } \\
\text { clínico. }\end{array}$ \\
\hline Estágio 0 & $\begin{array}{l}\text { Sem evidência de osso necrótico, porém com achados clínicos } \\
\text { inespecíficos, alterações radiográficas e sintomas. }\end{array}$ & $\begin{array}{l}\text { Manejo sistêmico com uso de analgésicos e } \\
\text { antibióticos. }\end{array}$ \\
\hline Estágio 1 & $\begin{array}{l}\text { Osso necrótico exposto ou fístula em pacientes assintomáticos e sem } \\
\text { evidência de infecção. }\end{array}$ & $\begin{array}{l}\text { Enxaguatório bucal antibacteriano, seguimento } \\
\text { clínico, orientações ao paciente, revisão para } \\
\text { ver se é indicado continuar com as } \\
\text { medicações. }\end{array}$ \\
\hline Estágio 2 & $\begin{array}{l}\text { Osso necrótico exposto ou fistula, com dor e eritema em região do osso } \\
\text { exposto, com ou sem drenagem purulenta. }\end{array}$ & $\begin{array}{l}\text { Enxaguatório bucal antibacteriano, } \\
\text { antibióticos, debridamento cirúrgico } \\
\text { superficial para diminuir irritação tecidual e } \\
\text { controle de infecção, controle da dor. }\end{array}$ \\
\hline Estágio 3 & $\begin{array}{l}\text { Osso necrótico exposto ou fístula, dor, infecção ou outros } \\
\text { acometimentos: fratura patológica, extensão da ON até borda inferior da } \\
\text { mandíbula ou seio maxilar, fístula extraoral, comunicação buco sinusal. }\end{array}$ & $\begin{array}{l}\text { Enxaguatório bucal antibacteriano, antibióticos } \\
\text { e controle da dor, debridamento cirúrgico ou } \\
\text { ressecção para tratamento paliativo prolongado } \\
\text { da dor e infecção. }\end{array}$ \\
\hline
\end{tabular}

Fonte: Ruggiero et al. (2014) (adaptado).

As terapias conservadoras promovem a manutenção da higiene oral e consequente eliminação de patógenos bucais através do uso tópico de enxaguatórios bucais e terapia antibiótica sistêmica. Embora possa não promover o objetivo do fechamento da ferida cirúrgica de acordo com seu estadiamento, a alternativa pode trazer conforto sintomático a longo prazo (Sturrock et al., 2019; Vescovi et al., 2013). Porém devem ser descontinuadas mediante progressão da lesão, sintomatologia dolorosa presente ou se o paciente tiver a terapia com bifosfonatos descontinuada pelo oncologista em decorrência da osteonecrose (Atalay et al., 2011).

Além das condutas recomendadas pela AAOMS, temos também terapias coadjuvantes que vêm sendo utilizadas em larga escala e descritas pela literatura para manejo da osteonecrose dos maxilares como a Laserterapia. Sua utilização dentro da odontologia é bastante ampla, sendo usada para terapia de lesões em tecidos moles, por exemplo. O laser de baixa potência diante da osteonecrose dos maxilares age em duas etapas sendo a primeira a ativação dos osteoblastos para produção de matriz óssea e a segunda etapa baseia-se na diminuição da atividade dos osteoblastos por um meio fisiológico e estimulação dos osteoclastos para que através da sua atividade desenvolvam a reabsorção e consequente processo de remodelação óssea (Bicudo, 2015; Starling, 2018; Menezes et al., 2021).

A Terapia fotodinâmica (aPDT) consiste no uso de um corante fotoativo (fotossensibilizante) que será ativado pelo laser (luz com comprimento de onda específico). A terapia possui efeito antimicrobiano impedindo o desenvolvimento de micro-organismos específicos que possam alterar a microbiota da cavidade oral deixando-a favorável para o desenvolvimento de infecções, promovendo a antissepsia da ferida. A ativação do corante promove uma reação com a liberação de um tipo diferente de oxigênio, mais tóxico e de radicais livres oxigênio tóxico e capazes de destruir lipídeos, ácidos nucleicos e outros componentes celulares (Bicudo, 2015; Menezes et al., 2021; Ferreira et al., 2013).

É importante salientar que alguns fatores influenciam a eficácia da Terapia Fotodinâmica tais como: tipo e concentração de fotossensibilizador (o azul de metileno e azul de toluidina são corantes muito utilizados), microbiota, potência e comprimento de onda do laser e tempo de exposição da terapia. Estudos clínicos mostram resultados satisfatórios como cicatrização da ferida em mucosa, efeito analgésico, reparo alveolar pós exodontias em pacientes que fazem uso de bifosfonatos e até mesmo prevenção da osteonecrose (Bicudo, 2015; Menezes et al., 2021; Ferreira et al., 2013).

Outra alternativa é a ozonioterapia, que possui propriedades: antimicrobianas (com ação bactericida contra G+ e G-, fungicida e virucida), antiálgicas (devido ao fato de neutralizar mediadores neuroquímicos da dor) e anti-inflamatórias (pela metabolização e eliminação de mediadores da inflamação. Devido a isso, o uso da ozonioterapia traz bons resultados quando 
aplicadas direta ou indiretamente nas lesões de ONM promovendo melhor oxigenação da lesão e neoangiogênese. O fato de não apresentar efeitos colaterais e contraindicações torna terapia promissora para o tratamento da osteonecrose dos maxilares, sedo ainda necessários estudos que comprovem sua eficácia diante da patologia (Bicudo, 2015; Menezes et al., 2021; Kagami et al., 2018).

Estudos ainda são necessários para comprovar a eficácia da oxigenação hiperbárica, como tratamento único (sem demais coadjuvantes) para a osteonecrose dos maxilares. O tratamento atua aumentando os níveis de oxigênio no sangue trazendo consequentemente benefícios para a cicatrização da ferida operatória, mobilização de células de estamina, reduzindo o efeito de supressão do turnover ósseo, reduzindo o edema e diminuindo os níveis de dor. Além disso, o uso da oxigenação hiperbárica para tratamento da osteonecrose dos maxilares induzida por bifosfonatos pode ser justificada pelo seu efeito angiogênico aumentando a vascularização que é prejudicada devido a patologia. Além disso algumas limitações impedem a terapia com oxigenação hiperbárica, como pacientes com metástases ósseas, presença de câncer devido a malignidade e claustrofóbicos por exemplos (Bicudo, 2015; Starling, 2018; Menezes et al., 2021).

A terapia cirúrgica como tratamento da osteonecrose dos maxilares é bem elucidada pela literatura, porém, requer planejamento minucioso e conhecimento de técnicas afim de evitar agravos ou recidivas. Dentre as técnicas cirúrgicas destacamos a curetagem (maior risco de recidiva), sequestrotomia ou desbridamento, saucerização e mandibulectomia em casos mais severos de osteonecrose (Bicudo, 2015; Menezes et al., 2021; Kagami et al., 2018).

Deve ser realizada medicação pré-operatória 3 dias antes do procedimento com Amoxicilina (2 gramas ao dia) e metronidazol (1 grama ao dia) devendo ser continuada por até 2 semanas após o procedimento cirúrgico. Durante o procedimento cirúrgico alguns cuidados devem ser tomados como a remoção total do tecido necrótico. Para isso, alguns cirurgiões usam como parâmetro para identificação de osso sadio o sangramento ósseo, porém, se tratando de osteonecrose isso não pode ser levado em consideração devido ao fato de que estudos recentes mostram que a vitalidade óssea nem sempre está correlacionada a esse parâmetro, sendo a fluorescência uma técnica muito utilizada em cirurgias de osteonecrose a fim de distinguir osso necrótico de osso vital. A técnica baseia-se administração de antibióticos com propriedades fluorescentes como a tetraciclina e seus derivados, antes do procedimento cirúrgico. Esses antibióticos incorporam no osso, que sendo vital que assume uma coloração verde fluorescente quando exposto a uma luz com comprimento de onda específico (525 a $540 \mathrm{~nm}$ ), diferente do osso necrótico que não apresenta fluorescência (Vescovi et al., 2012; Atalay et al., 2011; Rugani et al., 2013; Vescovi et al., 2013; Brozoski et al., 2012)

Além disso, a remodelação de arestas ósseas deve ser realizada a fim de evitar trauma em mucosas e consequente recidiva da lesão necrótica, após é necessária a irrigação com solução antibacteriana e sutura livre de tensão para coaptação de bordas sendo executada de preferência com fio de sutura reabsorvível (Vescovi et al., 2012; Atalay et al., 2011; Rugani et al., 2013; Vescovi et al., 2013). A avaliação pós operatória deve ser periódica e a avaliação radiográfica deve ser realizada após 6 meses. Reintervenções cirúrgicas devem ser realizadas após 3 meses caso necessidade. A literatura mostra bons resultados quando temos como coadjuvantes a terapia cirúrgica as seguintes terapias: terapia farmacológica (associada ao uso de antibióticos), laserterapia de baixa potência (LLLT), ozônioterapia e membranas biológicas como plasma risco em plaquetas (PRP) e fibrina rica em plaquetas e leucócitos (Bicudo, 2015; Menezes et al., 2021; Khan et al., 2015; Kagami et al., 2018).

\section{Discussão}

O bifosfonato vem sendo vastamente aplicado em terapias de pacientes que apresentam desordens no metabolismo ósseo que ocasionam a reabsorção exagerada e consequente diminuição da qualidade óssea. Devido a influência do medicamento sobre o metabolismo, observamos a supressão do turnover ósseo através da diminuição da atividade 
osteoclástica, fato que contribui para o surgimento da osteonecrose dos maxilares. (Castro et al., 2015; Fernandes et al., 2005; Chaves et al., 2018; Sousa et al., 2008; Mendes, 2017; Fahd, 2020; Santos et al., 2021; Parrião et al., 2021).

De etiologia pouco elucidada, mas, muito abordada em literatura, acredita-se que alguns fatores em especial podem contribuir para o desenvolvimento desta condição, entre eles: características específicas da droga como a supressão do turnover ósseo e o efeito antiangiogênico somado a fatores como a dosagem dos medicamentos, via de administração, tempo de uso e patologia a ser tratada e a presença de fatores de risco locais e sistêmicos (Bicudo, 2015; Ruggiero et al., 2014; Couto, 2018; Sousa et al., 2008; Santos et al., 2021; Pinto Junior et al., 2017; Carlessi et al., 2017; Santos, et al., 2020; Parrião et al., 2021; Spezzia, 2019; Carneiro et al., 2020).

Os critérios de diagnósticos elencados pela AAOMS: área de exposição óssea ou osso que possa ser sondado através de fístula intra ou extraoral de surgimento espontâneo ou induzido por cirurgias em região maxilofacial que persista por 8 ou mais semanas; tratamento atual ou prévio com medicações antirreabsortivas ou antiangiogênicas e ausência de histórico de radioterapia em região maxilofacial e de doença metastática óbvia na região dos maxilares (Ruggiero et al., 2014), são citados em grande parte dos artigos selecionados.

Como exames complementares de diagnóstico, as radiografias panorâmicas e periapicais diante da presença de osteonecrose dos maxilares em estágios iniciais podem não revelar alterações muito significativas necessitando de exames imaginológicos tridimensionais como a tomografia computadorizada de feixe cônico que oferecem como vantagem a maior qualidade de imagem e possibilidade de identificação e definição das margens da lesão necrótica determinado sua extensão e consequentemente, sua gravidade (Bicudo, 2015; Olutayo et al., 2010). Porém, em estágios mais avançados como 2 e 3, as radiografias panorâmicas e periapicais podem apresentar como características de alteração imagens radiolúcidas difusas, aumento do espaço do ligamento periodontal, espaços alveolares crônicos e presença de esclerose óssea, por exemplo (Bicudo, 2015; Olutayo et al., 2010; Arce et al., 2009; Spezzia, 2019).

É consenso que a prevenção para pacientes que iniciarão, estão ou que já concluíram a terapia com medicações antirreabsortivas como os bifosfonatos é indispensável (Bicudo, 2015; Wan et al., 2020; Sturrock et al., 2019). Para isso, a avaliação do risco de desenvolvimento da osteonecrose dos maxilares se faz necessária, permitindo melhor planejamento do tratamento a ser realizado e diminuindo as chances de desenvolvimento. É feita através da anamnese com coleta de informações detalhadas, exame clínico e exames complementares minuciosos para posterior e classificação de risco e adequação de meio bucal, com a remoção de fontes de trauma e infecções dento-alveolares (Gonzales et al., 2019; Spezzia, 2019; Carneiro et al., 2020; Meneguini et al., 2017).

Em casos de ocorrência, o tratamento tem como principal objetivo o fechamento da ferida cirúrgica e controle da sintomatologia dolorosa e de infecções secundárias. Este, deve ser realizado através do estadiamento da lesão necrótica e das condições sistêmicas atuais do paciente, dito uma vez que pacientes em tratamento oncológico por exemplo, podem necessitar de ocasiões específicas para o manejo da osteonecrose. É valido salientar que a terapia deve ser remanejada toda vez que o paciente apresentar progressão da condição. Um protocolo de condutas recomendadas para tratamento da complicação foi elaborado pela AAOMS, elegendo a conduta indicada de acordo com o estágio e pode ser realizado em conjunto com algumas terapias como a terapia fotodinâmica, laserterapia e ozonioterapia. A utilização de fluorescência durante procedimentos cirúrgicos com a finalidade de distinguir osso saudável de osso necrótico, tem se mostrado muito eficiente e preserva o osso viável. Com isso, temos protocolos, terapias adjuvantes e técnicas que apresentam grande sucesso em atingir o objetivo principal destacado pelos autores (Bicudo, 2015; Ruggiero et al., 2014; Sturrock et al., 2019; Oliveira et al., 2014; Spezzia, 2019; Vilela-Carvalho et al., 2018; Khan et al., 2015; Fleisher et al., 2010). 


\section{Conclusão}

A osteonecrose dos maxilares induzida por bifosfonatos corresponde a uma complicação de origem multifatorial que ainda não é completamente elucidada pela literatura e não apresenta uma incidência bem definida devido ao fato de que demais patologias da cavidade oral apresentam características semelhantes a osteonecrose, dificultando o diagnóstico e consequentemente o tratamento.

Destaca-se a importância da elaboração de protocolos efetivos para pacientes que iniciarão, estão ou já concluíram a terapia com estes fármacos devido ao poder acumulativo dos bifosfonatos no organismo durante anos, sendo dessa forma necessária a avaliação do risco de desenvolvimento e do mapeamento de risco, respectivamente.

É evidente que a adequação de meio bucal e eliminação de possíveis fontes de trauma, focos de infecção e elaboração de plano de tratamento em conjunto com a equipe elegendo o período mais favorável para realização de determinados procedimentos são indispensáveis para a manutenção da saúde oral e sistêmica do paciente em tratamento com drogas antirreabsortivas como os bifosfonatos.

O uso de exames complementares de imagem como as radiografias panorâmicas e tomografias computadorizadas mostram-se grandes auxiliadores do diagnóstico em especial em estágios mais avançados devido a presença de características clínicas como espaços alveolares vazios após as extrações, aumento do correspondente ao ligamento periodontal entre as raízes e alterações escleróticas. O CTX-1 (marcador sérico de reabsorção óssea) possui variação que pode ser causada pela terapia com os bifosfonatos ou até mesmo pela doença em tratamento sendo então ineficaz para a avaliação de risco de desenvolvimento da osteonecrose.

O tratamento da complicação em questão é complexo e objetiva o fechamento da ferida necrótica e protocolos terapêuticos adjuvantes são descritos em literatura apresentando índices de sucesso como a laserterapia, terapia fotodinâmica, ozonioterapia, sendo ainda necessário o aprofundamento em estudos relacionados a Oxigenação hiperbárica como tratamento adjuvante na osteonecrose dos maxilares. É relevante reforçar que toda e qualquer terapia deve ser repensada diante da progressão da osteonecrose.

Levando em consideração a relevância do tema, estima-se que novos trabalhos sejam realizados para que este assunto seja melhor divulgado e entendido entre os profissionais. Conclui-se por fim que o acompanhamento periódico e a manutenção da saúde bucal por um cirurgião-dentista e a inserção deste profissional em uma equipe multidisciplinar durante a terapia com os bifosfonatos é o ponto chave para estes pacientes na tentativa de diminuir a ocorrência desta complicação garantindo assim saúde, assistência e bem-estar.

\section{Agradecimentos}

Agradecemos a todos que direta ou indiretamente contribuíram para a realização e sucesso do artigo.

\section{Referências}

Arce, K., Assael, L. A., Weissman, J. L. \& Markiewicz, M. R. (2009) Imaging Findings in Bisphosphonate-Related Osteonecrosis of Jaws. Journal of Oral and Maxillofacial Surgery, 67(5), 75-84.

Atalay, B., Yalcin, S., Emes, Y., Aktas, I., Aybar, B., Issever, H., Mandel, N. M., Cetin, O. \& Oncu, B. (2011) Bisphosphonate-related osteonecrosis: laserassisted surgical treatment or conventional surgery? Lasers Med Sci., 26, 815-823.

Bicudo, L. L. (2017) Osteonecrose dos Maxilares: prevenção e manejo. Palestra. II Congresso Brasileiro de Odontologia Hospitalar e Intensiva. Curitiba, SP.

Bicudo, L. L. (2015) Osteonecrose dos maxilares - cuidados e orientações. Conferência. $5^{\circ}$ Congresso da Faculdade de Odontologia de Araçatuba.

Brozoski, M. A., Traina, A. A., Deboni, M. C. Z., Marques, M. M. \& Naclério-Homem, M. G. (2012) Osteonecrose maxilar associada ao uso de bisfosfonatos. Rev Bras Reumatol., 52(2), 260-270. 
Carlessi, V. \& Maragno, A. C. (2017) Pesquisa de ocorrência de osteonecrose em pacientes oncológicos submetidos ao tratamento com bifosfonatos de um hospital do sul de Santa Catarina. (Monografia, Curso de Odontologia da Universidade do Extremo Sul Catarinense, UNESC). http://repositorio.unesc.net/handle/1/5359.

Carneiro, L. K. De A., Fernandes, V. P., \& Conceição, L. S. da. (2020) Osteonecrose associada à cirurgia odontológica decorrente do uso de bifosfonatos: revisão de literatura. FACIT Business and Technology Journal, 3(19), 72-82. https://jnt1.websiteseguro. com/index.php/JNT/article/view/727.

Castro, L. F., Silva, A. T. A., Chung, M., Ferreira, A. G. \& Ferreira, E. I. (2004) Bifosfonatos (Bfs) como transportadores osteotrópicos o planejamento de fármacos dirigidos. Quim. Nova, 27(3), 456-460.

Chaves, R. A. C., Queiroz, T. P., Faloni, A. P. S., Órfão, A. M. A. \& Betoni-Júnior, W. (2018) Bifosfonatos e denosumabes: mecanismos de ação e algumas implicações para a implantodontia. Revista Brasileira Multidisciplinar-ReBraM, 21(2).

Costa, J. S. (2020) Tratamento para osteonecrose dos maxilares induzidos por drogas antirreabsortivas uma revisão de literatura. (Artigo, Curso de Odontologia. Escola Bahiana de Medicina e Saúde Pública). Salvador, BH.

Couto, E. D. S. Z. (2018) A terapia com bifosfonatos e a osteonecrose dos maxilares. Clube de autores.

Dal-Prá, K. J., Lemos, C. A. A., Soubhia A. M. P., Okamoto, R. \& Pellizzer, E. P. (2016) Eficácia do exame telopeptídeo C-terminal na previsão do desenvolvimento de osteonecrose dos maxilares relacionada ao uso de bisfosfonatos: revisão sistemática. Proceedings of the $1^{\circ}$ Encontro de Revisão Sistemática elou Meta-Análise do Programa de Pós-Graduação em Odontologia - FOA/UNESP, 5.

Fahd, L. B. (2020) Osteonecrose dos maxilares associada ao uso de Bifosfonatos: relato de caso clínico. Rev. Mult. Psic., 8(1).

Fernandes, C., Leite, R. S. \& Lanças, F. M. (2005) Bisfosfonatos: síntese, análises químicas e aplicações farmacológicas. Quim. Nova, 28(2), 274-280.

Ferreira, S., Mariano, R. C., Garcia Junior, I. R. \& Pellizer, E. P. (2013) Ozônioterapia no controle da infecção em cirurgia oral. Revista Odontológica de Araçatuba, 34(1), 36-38.

Fleisher, K. E., Welch, G., Kottal, S., Craig, R. G. Ms, D. S. \& Glickman, R. S. (2010, out) Predicting risk for bisphosphonate-related osteonecrosis of the jaws: CTX versus radiographic markers. Oral Radiol Endod., 110(4)

Gonzales, J. M. G. \& Azevedo, A. (2019) Osteonecrose mandibular induzida por bisfosfonatos. (Dissertação de Mestrado Integrado em Medicina Dentária, Instituto Universitário de Ciências da Saúde - CESPU, Gandra)

Kagami, H., Inoue, M., Kobayashi, A., Taguchi, A., Li, X. \& Yoshizawa, M. (2018) Issues with the surgical treatment of antiresorptive agent-related osteonecrosis of the jaws. Oral Dis, 24, 52-56.

Khan, A. A., Morrison, A., Hanley, D. A., Felsenberg, D., Mccauley, L. K. \& O’Ryan, F. (2015) Diagnosis and management of osteonecrosis of the jaw: a systematic review and international consensus. J Bone Miner Res, 30(1), 3-23.

Lazarovici, T. S., Mesilaty-Gross, S., Vered, I., Pariente, C., Kanety, H., Givol, N., Yahalom, R., Taicher, S. \& Yarom, N. (2010) Serologic Bone Markers for Predicting Development of Osteonecrosis of the Jaw in Patients Receiving Bisphosphonates. American Association of Oral and Maxillofacial Surgeons J Oral Maxillofac Surg, 2241-2247.

Marx, R. E. (2003) Pamidronate (Aredia) and zoledronate (Zometa) induced avascular necrosis of the jaws: a growing epidemic. Journal of oral and maxillofacial surgery, 61(9), 1115-1117.

Mendes, J. M. (2017) Bifosfonatos: aspetos de segurança. (Tese, Curso de Mestrado em Ciências Farmacêuticas, Universidade Fernando Pessoa, Porto).

Meneguini, L. S., Nogueira, G. T., Tavares, L. B., Albergaria, B., Veloso, T. R. G., Alencar, C. O. de \& Bianchi, P. R. (2017) Avaliação de fatores de risco para osteonecrose em pacientes usuários de Bifosfonatos no HUCAM/UFES. Braz J Periodontol, 27.

Menezes, I. L., Sales, J. M., Targino, M. L. de M., Vasconcelos, R. G. \& Marinho, S. A. (2021) Terapia fotodinâmica no tratamento de osteonecrose mandibular por Bisfosfonatos: uma revisão. Brazilian Journal of Health Review, 4(1), 2652-2665.

Oliveira, M. A. de, Martins, F. M., Asahi, D. A., Santos, P. S. da S. \& Gallottini, M. (2014) Osteonecrose induzida por bisfosfonatos: relato de caso clínico e protocolo de atendimento. Arq Med Hosp. Fac. Cienc. Med Santa Casa São Paulo, 59(1), 43-48.

Oliveira, M. E. de F. S., Líbera, J. D. L., Lucatto, N. F., Carvalho, M. M. de, Oliveira, L. F., Santana, M. R. de O., Villela-Rosa, A. C. M. \& Simonato, L. E. (2020) Aspectos radiográficos da osteonecrose em maxilares por bisfosfonatos. Rev Odontol UNESP. 49, $1807-2577$.

Olutayo, J., Agbaje, J. O., Jacobs, R., Verhaeghe, V., Velde, F. V. \& Vinckier, F. (2010, abr/jun) Bisphosphonate-Related Osteonecrosis of the Jaw Bone: Radiological Pattern and the Potential Role of CBCT in Early Diagnosis. J Oral Maxillofac Res, 1(2), 9.

Parrião, E. V., Silva, F. M. F. da, Carvalho, N. A. de, Cardoso, J. C. da S., Góes, R. W. L., Dietrich, L. \& Costa, M. D. M. de A. (2020) Bisfosfonatos e Osteonecrose Maxilar. Scientia Generalis, 1, 59.

Pexe, M., Curi, M. M., Costa, B. E., Koga, D. H. \& Cardoso, C. L. (2018) Estudo retrospectivo da osteonecrose dos maxilares associada ao uso de bisfosfonatos sem exposição óssea clínica. Rev. Salusvita, 37(4), 867-878, https://pesquisa.bvsalud.org/gim/resource/en/biblio-1050817? src=similardocs.

Pinto Junior, A. A. C., Macedo, L. M., Moreira, L. I. R., Alves, J. de F. C. S. \& Lacerda, J. C. T. de. (2017) Osteonecrose dos Maxilares Associada ao uso de Bisfosfonatos. Rev. Cir. Traumatol. Buco-Maxilo-Fac., 17(1), 40-45.

Rugani, P., Truschnegg, A., Acham, S., Kirn-Bauer, B. \& Jakse, N. (2013) Use of photodynamic therapy in treatment of bisphosphonate-related osteonecrosis of the jaws: literature review and case series. J Anal Bioanal Tech, 1(6). 
Ruggiero, S. L., Dodson, T. B., Fantasia, J., Gooddav, R., Aghaloo, T., Mehrotra, B. \& O’Ryan, F. (2014) American Association of Oral and Maxillofacial Surgeons Position Paper on Medication-Related Osteonecrosis of the Jaw - 2014 Update. American Association of Oral and Maxillofacial Surgeons J Oral Maxillofac Surg.,72, 1938-1956.

Ruggiero, S. L., Dodson, T. B., Assael, L. A., Landesberg, R., Marx, R. E. \& Mehrotra, B. (2009) American Association of Oral and Maxillofacial Surgeons Position Paper on Bisphosphonate-Related Osteonecrosis of the Jaws-2009 Update. J Oral Maxillofac Surg., 67, 2-12.

Santos, G. M. R. dos \&, Ferreira Neto, M. \& D’Almeida. (2021) Relação da Osteonecrose dos maxilares com o uso dos Bisfosfonatos: Uma Revisão Integrativa. Rev. Mult. Psic, 15(56), 214-224.

Santos, W. B. do, Pereira, R. da S., Gonçalves, V. C. de B., Nascimento, S. V. do, Silva, A. H. de M., Costa, A. M. G., Melo, M. W. V., Correia Neto, I. J. \& Kalinin, Y. (2020) Osteonecrose dos Maxilares associada ao uso crônico de bisfosfonatos: relato de caso. Revista Eletrônica Acervo Saúde.

Sousa, F. R. N. \& Jardim Junior, E. G. (2008) Osteonecrose Associada com o uso dos Bifosfonatos. Pesquisa Brasileira em Odontopediatria e Clínica Integrada, 8(3), 375-380.

Spezzia, S. (2019) Implicações odontológicas do emprego dos bisfosfonatos: osteonecrose no complexo ósseo maxilo-mandibular. RCO., 3(2), 27-34.

Starling, I. R. N. (2018) Tratamento de osteonecrose extensa associada ao uso de anti reabsortivos: relato de caso e revisão de literatura. (Monografia, Curso de Especialização em Cirurgia e Traumatologia Bucomaxilo-facial, Faculdade de Odontologia. Universidade Federal de Minas Gerais, Belo Horizonte).

Sturrock, A.; Preshaw, P. M.; Hayes, C.; \& Wilkes, S. (2019). Perceptions and attitudes of patients towards medication-related osteonecrosis of the jaw (MRONJ): a qualitative study in England. BJM Open, 9. 1-7.

Vescovi, P., Meleti, M., Merigo, E., Manfredi, M., Fornaini, C., Guidotti, R. \& Nammour, S. (2013) Case series of 589 tooth extractions in patients under bisphosphonates therapy. Proposal of a clinical protocol supported by Nd: YAG low-level laser therapy. Med Oral Patol Oral Cir Bucal, 1(18), 680-685.

Vescovi, P., Merigo, E., Meleti, M., Manfredi, M., Guidotti, R. \& Nammour, S. (2012) Bisphosphonates-related osteonecrosis of the jaws: a concise review of the literature and a report of a single-center experience with 151 patients. J Oral Pathol Med, 41(3), 214-221.

Vilela-Carvalho, L. N., Duarte, N. T., Figueiredo, M. A. \& Ortega, K. L. (2018) Osteonecrose dos maxilares relacionada ao uso de medicações: Diagnóstico, tratamento e prevenção. Rev. CES Odont., 31(2), 48-63.

Wan, J. T., Sheeley, D. M., Somerman, M. J., \& Lee, J. S. (2020) Mitigating osteonecrosis of the jaw (ONJ) through preventive dental care and understanding of risk factors. Bone Research. 